\title{
Differential payoff-loss in probability-learning and decision-making
}

J. RONALD GENTILE AND LOWELL M. SCHIPPER

THE PENNSYLVANIA STATE UNIVERSITY

Payoffs were differentially manipulated in a modified factorial arrangement to determine the effects on the probability-learning (PL) of two independent events and a subsequent decision-making (DM) task based on the PL. Results were consistent with previous studies using an "absolute" method of incentive distribution. However, differentially weighting the low-probability event appeared to effect early learning and subsequent decision behavior.

Incentive magnitude has been shown to have different effects on learning depending on whether an "absolute" method (where $S$ receives a single incentive level) or a "differential" method (where $S$ receives more than one incentive level) is used. Experiments on both animals (Pubols, 1960) and humans (Lipkin et al, 1965; Myers et al, 1963; and Schnorr et al, 1965) show magnitude of incentive to have no effect on timeindependent measures of learning when an "absolute" method is used, whereas incentive effects have been shown in experiments using a "differential" method.

In the Lipkin and Schnorr studies the within-Ss treatment ("differential" method) was a probabilitylearning (PL) task in which the events to be predicted were worth either 10 cents or 1 cent randomly arranged and both pay-off levels occurred with each event, $\pi_{1}=.60$ and $\pi_{2}=.40$, equally often. In contrast, the present study was an "absolute" design in which the consistent effects of differentially paying-off two independent events with different probabilities were investigated in a modified factorial arrangement. Further, the effects of these differential pay-offs were examined in a subsequent decision-making (DM) situation in which knowledge of results was not given.

Method

The display panel consisted of two green lights arranged horizontally about $8-1 / 2$ in. apart with a single red light about $2-1 / 2$ in. between and below them. The occurrence or nonoccurrence of each light was controlled using Western Union Tape Programmers.

In the PL part of the experiment Ss saw one of two green lights come on for $5-1 / 2 \mathrm{sec}$. and, if it were to occur on that trial, a red light which occurred during the last $3 \mathrm{sec}$. of the green light. One of the green lights was programmed to be followed randomly by the red event light on $80 \%$ of the trials on which it occurred; the other was followed randomly on $20 \%$ of the trials. The left-right positions of the green lights were randomly selected by sessions. There were 300 training trials, 150 on each green light with an intertrial interval of 2 sec.
In the DM phase of the experiment the red light was covered to prevent feedback. Ss were then given 30 mixed trials, 10 on each of the green lights separately (as in PL) and 10 with the two green lights occurring simultaneously.

There were six experimental treatments: (1) PennyPenny (P-P), in which Ss won a penny each time they were correct and lost a penny each time they were incorrect in predicting the occurrence or nonoccurrence of the event light; (2) Nickel-Nickel (N-N), in which Ss won or lost nickels instead of pennies; (3) PennyNickel $(\mathrm{P}-\mathrm{N})$, in which Ss won or lost a penny on the $80 \%$ predictor light and won or lost a nickel on the $20 \%$ light; (4) Nickel-Penny (N-P), in which Ss won or lost a nickel on the $80 \%$ light and a penny on the $20 \%$ light; (5) No Pay-off, in which Ss were simply told to "try to be correct as often as possible", and (6) Utility of Variability ( $U$ of $V$ ), in which Ss manipulated red plastic discs (having no monetary value) in the same way coins were manipulated in treatments 1 through 4 . Treatment (6) was a control for the assumed maximizing effect of the "kinesthetic enrichment" (Siegel et al, 1964) of the manipulation of the coins, while treatment (5) was a control for value to measure the effect of the increased pay-off treatments.

Ss, who were 74 male introductory psychology students at The Pennsylvania State University, were nonsystematically selected by experimental session to receive one of the 6 treatments, and were run in groups of 2 to 6 at a time (except for $1 \mathrm{~S}$ ) in a total of 19 sessions with the same $E$ for all sessions. Four treatments had $12 \mathrm{Ss}$; the other 2, 13.

All responses were made by pushing a yes or no button on an individual response box and were recorded and summed remotely by a small computer. Ss were to predict whether the red light would follow whichever green light occurred. During PL if S were correct, he took an appropriate coin from the bank and placed it in his cash box. If $\mathrm{S}$ were incorrect, he transferred a coin from his cash box to the bank. Ss were told that they could keep all winnings over and above the original stake。

\section{Results}

1. There were no consistent differences among the pay-off groups ( 1 through 4) in final level of responding to either event light in the PL or DM phases of the experiment.

2. There were no real differences between the No Pay-off and the $U$ of $V$ groups either in learning the probabilities of the 2 lights or in the DM phase (when 
the red event light was covered). However, in all comparisons the differences were in the direction predicted by Siegel et al.

3. Greater maximization-that is, lower group per cent yes responses-occurred in the pay-off groups than in the no-pay-off groups in PL on the $20 \%$ light $(p<.005, t=2.982, d f=72,1$-tailed, after significant $F$ in anova), but there was no overall difference among sroups on the $80 \%$ light $(p>.10, F<1.00, \mathrm{df}=5 / 68)$.

The general negative results in PL in these betweentreatment comparisons are consistent with the findings reported in the introduction despite the differential payoffs of the events within treatments.

t. When both predictor lights occurred simultaneously in $\mathrm{DM}$, requiring $\mathrm{Ss}$ to combine in some way the probabilities they learned to associate with each light individually, there were no statistically reliable differences in numbers of yes responses between pay-off and no-pay-off (Figure 1).

\section{Discussion}

A possible interesting departure from the overwhelming "no differences" results occurred in a comparison of the $\mathrm{P}-\mathrm{N}$ and $\mathrm{N}-\mathrm{P}$ treatments' early learning. If this two-light PL situation is considered as a discrimination task, subtracting per cent yes responses on the $20 \%$ light from per cent yes response on the $80 \%$ light for successive blocks gives acquisition curves of discrimination between the two lights. Figure 2 suggests that differential monetary weighting of high- and low-probability predictors has its effect mainly on the rate of discriminating between these predictors and not on the final level of responding. Emphasizing the $20 \%$ light, as in the P-N treatment,

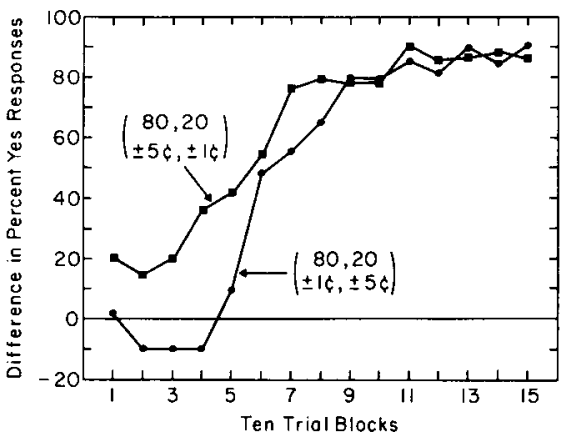

Fig, 2. Differences in learning to discriminate between $\pi_{1}=.80$ and $\pi_{2}=.20$ in penny-nickel and nickel-penny treatments.

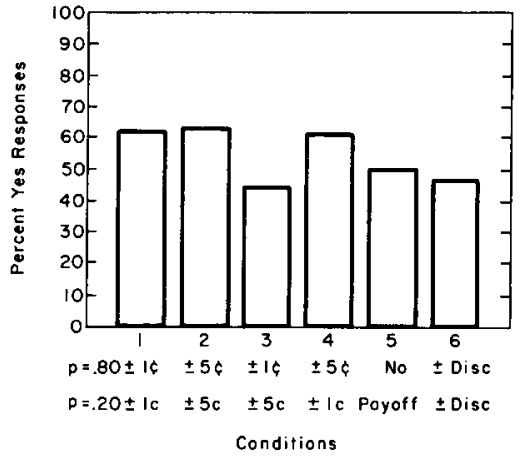

Fig. 1. Percentages of yes responses for the $.80-.20$ pattern in decision-making.

depresses the discrimination initially so that Ss respond with more yes responses to the $20 \%$ light than to the $80 \%$ light. Emphasizing the $80 \%$ light, as in the N-P treatment, appears to enhance early discrimination. The main difference in learning rate is for the $80 \%$ light and disappears after approximately 80 trials.

Finally, it is interesting that the $\mathrm{P}-\mathrm{N}$ treatment, whose early acquisition rate on the $80 \%$ light is retarded in comparison to the $\mathrm{N}-\mathrm{P}$ treatment, responded with fewer yes responses to the 80-20 combination (see Figure 1) in the DM phase $(p<.05, t=1.863, \mathrm{df}=23$, 1-tailed). It appears that weighting the low-probability light in PL has a depressing effect on the subsequent DM. An explanation of this relationship between the retarded early PL and the depressed DM behavior of the P-N treatment is not, however, immediately obvious.

\section{References}

Lipkin, S. G., Schnorr, J. A., Suydam, M. M.. \& Myers, J. L. Effects of risk as a within-subject variable in probability learning. Psychon. Sci., 1965, 2, 145-146.

Myers, J. L., Fort, J. G., Katz, L., \& Suydam, M. M. Differential monetary gains and losses and event probability in a two-choice situation. J. exp. Psychol., 1963, 66, 521-522.

Pubols, B. H., Jr. Incentive magnitude, learning, and performance in animals. Psychol. Bull., 1960, 57, 89-115.

Schnorr, J. A., Lipkin, S. G., \& Myers, J. L. Incentive contrast in probability learning. unpublished.

Siegel, S., Siegel, A. E., \& Andrews, J. M. Choice, strategy, and utility. New York: McGraw-Hill, 1964.

\section{Note}

1. This investigation was supported by a Public Health Sarvice Research Fellowship 2F1-MH-21, 734-02 from the National Institute of Mental Health to the senior author. Major components of the apparatus were supplied by The Boeing Company of Seattle, Washington on arrangement with the junior author. 